•编者按・

\title{
生态系统红色名录：进展与挑战
}

\author{
马克平 ${ }^{*}$ \\ (中国科学院植物研究所植被与环境变化国家重点实验室, 北京 100093)
}

\section{Red List of Ecosystems (RLE): progress and challenges}

\section{Keping $\mathrm{Ma}^{*}$}

State Key Laboratory of Vegetation and Environmental Change, Institute of Botany, Chinese Academy of Sciences, Beijing 100093

世界自然保护联盟(IUCN)在物种受威胁程度 评估标准的制订与应用方面具有权威性, 其不断更 新并发布的全球物种红色名录 (http://www.iucnredlist.org/)被广泛采用, 作为生物 多样性保护的重要参考。经过半个多世纪的努力, 物种红色名录项目取得了重要进展, 成为IUCN最 有影响力的标志性工作。主要体现在三个方面: (1) 为目标明确、过程透明且结果可重复的物种生存风 险评估建立了定量的评估标准; (2)从仅包括受威胁 物种的红色名录扩展到也包括未受威胁物种的不 断增长的物种数据库; (3)在不同国家和地区以及不 同生物类群中得到广泛应用(Rodríguez et al, 2012)。 IUCN 全球物种红色名录已经包括86,313 个物种, 其 中24,307种(28.2\%)受到威胁(www.iucnredlist.org/)。 据不完全统计, 不同国家或区域的不同类群的物种 红色名录已经发布了数千个, 仅欧洲就有 3,500 个 之多(Rodríguez et al, 2012); 100多个国家发布了至 少 250 个不同类群的国家物种红色名录(Zamin et al, 2010)。中国的高等植物和脊椎动物红色名录分别于 2013年和2015年由环境保护部和中国科学院正式 联合发布(蒋志刚等, 2015)。

物种红色名录可以指示生物多样性受威胁的 程度, 但不全面, 需要有生态系统受威胁程度的评 估予以补充。第一届IUCN自然保护大会于1996年 在加拿大蒙特利尔召开, 生态系统管理委员会 (Commission on Ecosystem Management)首任主席 Edward Maltby博士提出建立IUCN生态系统红色名 录的建议。2000年约旦安曼的第二届世界自然保护
大会上再次讨论这个议题，希望参照物种红色名录 的做法建立生态系统红色名录的标准和程序 (Rodríguez et al, 2012)。2004年在泰国曼谷召开的第 三届世界自然保护大会上召开专题研讨会, 讨论生 态系统红色名录的评估方法, 会后进一步完善并开 始试行。评估的内容主要包括四个方面: 面积减少 并面临持续的威胁、面积快速变化、片断化加剧、 地理分布范围狭窄(Rodríguez et al, 2007)。2008年3 月, 生态系统红色名录等级和标准临时工作组在伦 敦开会, 讨论当时已经发表的4个评估方案并准备 了一份拟提交2008年10月在西班牙巴塞罗那召开 的第四届世界自然保护大会审议的提案。提案被大 会通过成为决议(IUCN Resolution 4.020), 决定开 始IUCN官方咨询程序, 以建立适用于局地、区域和 全球水平的生态系统状态评估标准, 提交将来召开 的自然保护大会审议通过。

经过近三年的多方努力, 第一个综合方案草案 于2010年正式发布(Rodríguez et al, 2011)。其核心思 想是生态系统风险是其组成物种及其相互作用以 及相关的生态过程风险的函数, 具体体现于 4 条标 准: (1)近期分布区和生态功能的下降; (2)历史上分 布区和生态功能总的下降; (3)分布区狭窄且丧失; (4)非常小的分布区。根据在5大洲 20 个国家举办的 17次大会和18次研讨会征集的反馈意见，生态系统 红色名录等级和标准不断修改完善。2012年9月在 韩国平昌召开的第五届IUCN自然保护大会通过决 议(5.055), 要求IUCN生态系统管理委员会和秘书 处进一步修改完善后提交IUCN理事会审议通过,

* 通讯作者 Author for correspondence. E-mail: kpma@ibcas.ac.cn 
开始推广施行(附录1)。

与物种受威胁程度评估相比, 生态系统状态评 估有优势也有劣势。优势在于: (1)生态系统比物种 能够更好地代表完整的生物多样性, 特别是考虑到 目前的全球物种红色名录包括的类群很不均衡的 状况时更是如此; (2)生态系统状态恶化比物种丧失 或灭绝更容易观察到; (3)生态系统水平的评估比物 种水平评估需要的时间短得多; (4)生态系统红色名 录可能指示即将发生物种旋浴灭绝的生境; (5)生态 系统评估的空间属性为保护和土地利用规划提供 了更为直接的支持。相比之下, 生态系统红色名录 面对的挑战也是很棘手的。主要包括: (1)生态系统 分类难以统一; (2)评估的基本单元难以界定; (3)生 态系统“灭绝”难以定义; (4)等级和标准的阈值难以 量化(Rodríguez et al, 2012; Boitani et al, 2015; Keith et al, 2015)。

2016年在美国夏威夷召开的第六届世界自然 保护大会对于生态系统红色名录而言具有特别重 要的意义。在这次大会上, 正式发布了生态系统红 色名录的等级和标准(附录2), 并举办了多个培训班 和研讨会等。生态系统红色名录评估设立了 8 个等 级: 崩溃 $(\mathrm{CO}$, Collapse)、极危(CR, Critically Endangered)、濒危(EN, Endangered)、易危(VU, Vulnerable)、近危(NT, Near Threatened)、无危(LC, Least Concern)、数据不足(DD, Data Deficient)和未评估 (NE, Not Evaluated)。除最严重的等级“崩溃”之外, 其他等级的名称与物种红色名录完全相同, 但评估 标准则完全不同。谭剑波等(2017)在本期发表的评 述文章中介绍了评估标准和方法, 从生境范围退化 (标准 $\mathrm{A}) 、$ 生境限制分布(标准B)、非生物环境退化 (标准C)、生物过程退化(标准D)和威胁定量分析(标 准E) 5 个方面做了比较清楚的介绍。同时, 对目前开 展生态系统红色名录项目面临的挑战做了分析, 认
为“当前IUCN生态系统红色名录评估标准及相应國 值的设定缺乏理论和数据的支撑, 在生态系统制 图、生态系统崩溃的概念以及实际操作层面均不够 清晰, 易导致在应用时主观地解读评估体系, 形成 不确定的结果”。

2017年4月生态系统红色名录研讨会在英国伦 敦召开, 重点讨论了全球生态系统分类原则和框 架，以及应用生态系统红色名录过程中遇到的问题 和可能的解决办法。目前, 有 35 个生态系统红色名 录项目已经完成(Burns et al, 2015)，25个项目正在 进行中，涉及到500个生态系统类型。南非完成了第 二次国家水平的评估，芬兰正在开展第二次评估。 全球生态系统红色名录工作总体进展良好，但也存 在挑战。主要有四个方面: (1)经费不足使得一些国 家或地区的生态系统受威胁状态评估工作不能开 展; (2)全球生态系统分类体系及其分布图亟待构 建; (3)生态系统过程与功能变化以及遥感影像的地 面核实数据不足; (4)评估结果在管理中的应用和影 响力需要加强。

中国科学院生物多样性委员会分别于 2011年 和2015年与IUCN合作在北京举办过两次生态系统 红色名录培训研讨会, 对于IUCN生态系统红色名 录项目在中国的推广起到了积极作用。中国生态系 统红色名录项目于2016年5月由环境保护部正式启 动, 主要分为森林、草地(荒漠)和湿地生态系统三个 部分，已经取得了一定的进展。面临的挑战与伦敦 研讨会总结的四个方面类似。总体而言，实现已有 数据的共享和保持与国际同行的沟通交流对于项 目的顺利完成至关重要。第一个中国生态系统红色 名录有望在两年内完成。

文中引用的文献见附录3 (http://www.biodiversity-science.net/fileup/PDF/2017160-3.pdf)。

\section{附录 Supplementary Material}

附录 1 IUCN 自然保护大会决议 5.055: 生态系统红色名录的完善

Appendix 1 IUCN WCC Resolution 5.055: Consolidation of the IUCN Red List of Ecosystems http://www.biodiversity-science.net/fileup/PDF/2017160-1.pdf

附录 2 IUCN 生态系统红色名录等级和标准的应用指南

Appendix 2 Guidelines for the application of IUCN Red List of Ecosystems Categories and Criteria http://www.biodiversity-science.net/fileup/PDF/2017160-2.pdf

附录3 参考文献 Appendix 3 References

http://www.biodiversity-science.net/fileup/PDF/2017160-3.pdf 


\section{附录3 参考文献}

Boitani L, Mace GM, Rondinini C (2015) Challenging the scientific foundations for an IUCN Red List of Ecosystems. Conservation Letters, 8, 125-131.

Burns EL, Lindenmayer DB, Stein J, Blanchard W, McBurney L, Blair D, Banks SC (2015) Ecosystem assessment of mountain ash forest in the Central Highlands of Victoria, south-eastern Australia. Austral Ecology, 40, 386-399.

Jiang ZG, Qin HN, Liu YN, Ji LQ, Ma KP (2015) Protecting biodiversity and promoting sustainable development: in memory of the releasing of Catalogue of Life China 2015 and China Biodiversity Red List on the International Day for Biological Diversity. Biodiversity Science, 23, 433-434. (in Chinese) [蒋志刚，覃海宁，刘忆南，纪力强，马克平 (2015) 保护生物多样性, 促进可持续发展一一纪念《中国 生物物种名录》和《中国生物多样性红色名录》发布. 生 物多样性, 23, 433-434.]

Keith DA, Rodriguez JP, Brooks T, Burgman MA, Edmund G. Barrow EG, Bland L, Comer PJ, Franklin J, Link J, McCarthy MA, Miller RM, Murray NJ, Nel J, Nicholson E, Oliveira-Miranda MA, Regan TJ, Rodriguez-Clark KM, Rouget M, Spalding MD (2015) The IUCN Red List of Ecosystems: motivations, challenges and applications. Conservation Letters, 8, 214-226.
Rodríguez JP, Balch JK, Rodríguez-Clark KM (2007) Assessing extinction risk in the absence of species-level data: quantitative criteria for terrestrial ecosystems. Biodiversity and Conservation, 16, 183-209.

Rodríguez JP, Rodriguez-Clark KM, Baillie J, Ash N, Benson J, Boucher T, Brown C, Burgess ND, Collen B, Jennings M, Keith DA, Nicholson E, Revenga C, Reyers B, Rouget M, Smith T, Spalding M, Taber A, Walpole M, Zager I, Zamin T (2011) Establishing IUCN Red List criteria for threatened ecosystems. Conservation Biology, 25, 21-29.

Rodríguez JP, Rodríguez-Clark KM, Keith DA, Barrow EG, Benson J, Nicholson E, Wit P (2012) IUCN Red List of Ecosystems. S.A.P.I.E.N.S 5.2: http://sapiens.revues.org/1286.

Tan JB, Li AN, Lei GB, Ma KP, Chen GK (2017) Research advances and challenges in the IUCN Red List of Ecosystems. Biodiversity Science, 25, 453-463. (in Chinese with English abstract) [谭剑波, 李爱农, 雷光斌, 马克平, 陈国科 (2017) IUCN 生态系统红色名录研究进展. 生物多样性, 25, 453-463.]

Zamin TJ, Baillie J, Miller R, Rodríguez JP, Ardid A, Collen B (2010) National red listing beyond the 2010 target. Conservation Biology, 24, 1012-1020. 